\title{
A case of locally advanced pancreatic cancer successfully resected after 14 months therapy with gemcitabine and Meriva ${ }^{\circledR}$.
}

\section{Caterina Soldà ${ }^{{ }^{*}}$ MD, Romeo Bardini ${ }^{2}$ Prof, Francesca Zingales ${ }^{2}$ MD, Davide Pastorelli ${ }^{1}$ MD}

\author{
${ }^{1}$ Rare Tumor Unit, Istituto Oncologico Veneto-IRCCS, via Gattamelata 64, 35128, Padova, Italy \\ ${ }^{2}$ Department of Surgery Oncology and Gastroenterology, University of Padova, via Giustiani 2, \\ 35128 Padova, Italy
}

caterina.solda@ioveneto.it, romeo.bardini@unipd.it, francesca.zingales@sanita.padova.it, davide.pastorelli@ioveneto.it

Keywords: pancreatic cancer, locally advanced, gemcitabine, curcumin, neoadjuvant.

\begin{abstract}
Pancreatic cancer (PC) is one of the leading cause of cancer death. Neoadjuvant therapy has been progressively used in the context of locally advanced/unresectable disease improve patients' outcome. New drugs combination are associated with increased response rate but at the expense of higher toxicities. We presented a case of a patient with locally advanced disease treated who could undergo surgery after 14 cycles of therapy with gemcitabine and curcumin conjugated with phospholipids (Meriva ${ }^{\circledR}$ ). Treatment was well tolerated without severe toxicities or impairment of quality of life. The patient is disease free at 9 month from surgery and 28 months from initial diagnosis and he still is in good clinical conditions. The combination of gemcitabine and Meriva ${ }^{\circledR}$ was effective in allowing radical surgery in a patient with locally advanced PC. Moreover, this results was obtained with minimally toxic regimen.
\end{abstract}

\section{Introduction.}

Despite recent advances in both systemic and local therapies, pancreatic cancer (PC) still constitutes fourth leading cause of cancer death in the European Union and the United States [1].

The most significant factor predicting long-term survival in PC is a radical resection (R0), which is unfortunately feasible in only about $20 \%$ of cases at diagnosis [2]. Patients with locally advanced pancreatic cancer (LAPC) and borderline resectable PC have about a 50\% chance for curative resection, as compared with stage I and II PC [3,4], mainly because of the high frequency of invasion of the retroperitoneal margin and/or the nervous plexus of the superior mesenteric artery.

Recent meta-analyses on neoadjuvant treatments of borderline resectable and LAPC [5] showed an increased rate of $\mathrm{R} 0$ resections without negative impact on postoperative mortality and morbidity as compared with those treated by upfront surgery.

Gemcitabine-based studies showed a 20\%-30\% response rate, with significantly superior results in favour of combination therapies versus Gemcitabine alone were subsequent found [6]. Unfortunately, no study had resection as an end point and the gain in response was obtained at the expense of increased toxicities. Curcumine, the main biologically active component of the Saffron (Curcuma longa L.), has been tested for its pharmacological properties. These studies have confirmed its remarkable anti-cancer properties, anti-inflammatory and antioxidant. The mechanisms of action for anti-tumor activity of curcumin can be roughly summarized as follows: a) inhibition of tumor cell proliferation, b) induction of apoptosis, c) inhibition of cell transformation from normal to tumor, d) inhibition of invasiveness and metastasis, e) suppression of inflammation [7].

In our Institution we conducted a phase II trial to evaluate the effect of adding an extract of curcumin conjugated with phospholipids (Meriva ${ }^{\circledR}$. Indena S.p.A. Viale Ortles 12, Milano/Italy) to gemcitabine as first line therapy for locally advanced or metastatic PC. 
Here we report the case of a patient with LAPC initially unresectable who obtained partial response to treatment after 14 months of therapy and could undergo surgical resection.

\section{Case presentation.}

We report the case of a 52-year-old Caucasian man with newly diagnosed, apparently resectable, lesion of the pancreatic head. His medical history was significant only for the presence of hypertension, successfully managed with medical therapy. No tobacco smoking, alcohol or substance abuse were reported. In February 2013 he underwent exploratory laparotomy. Despite the radiologic appearance, at the time of surgery, the involvement of aorta and mesenteric vessels was evident. Thus, considering the extent of disease, a palliative gastroenteroanastomosis was performed and the patient was referred to our oncologic unit to evaluate possible systemic approaches. At first evaluation, he presented in good clinical condition; physical examination was negative and blood tests were in the normal rage. Baseline CA $19-9$ was $70.2 \mathrm{kU} / \mathrm{L}$ (normal range $0,0-37,0)$. We proposed the patients to participate to a trial exploring the role of adding Meriva ${ }^{\circledR}$ ( $2 \mathrm{~g} /$ die continuously) to standard first line gemcitabine $\left(1000 \mathrm{mg} / \mathrm{m}^{2}\right.$ on days $1,8,15$ every 4 weeks), with radiological assessment every 3 cycles.

After signing the informed consent, in February 2013 he started chemotherapy with disease stability at subsequent evaluations. Treatment was well tolerated and regularly administered without interruption or toxicities, apart from grade 1 thrombocytopenia according to Common Terminology Criteria for Adverse Events v3,0 after the eighth cycle. Patients continued the same treatment at a dose of $75 \%$ until 14 cycles. Surprisingly, the CT scan of August 2014 documented the reduction of the pancreatic mass, which measured $12 \mathrm{~mm}$ in greatest diameter; Ca 19-9 was $47.9 \mathrm{kU} / \mathrm{L}$. A PETCT was then performed, which confirmed the absence of secondary lesions and only identified a rather insignificant cefalopancreatic dishomogeneity. Thus, after multidisciplinary discussion, in September 2014 the patient underwent new surgical exploration and a duodenocephalopancreasectomy was performed. The final pathology report documented a moderately differentiated (G2) adenocarcinoma, ypT3 ypN0 (0/23), with negative surgical margins (R0). The postoperative course was complicated by right ureter injury that required ureteroanastomosis and stent placement. Renal function tests showed no alterations.

After discharge, we decided to start maintenance therapy with Meriva ${ }^{\circledR}$. The last radiological assessment in May 2015 was still negative for disease recurrence and ureteral stent was removed.

\section{Discussion.}

PC still represent one the leading cause of cancer death. It remains clear that surgical resection is a prerequisite for achieving long-term survival and cure. In recent years the increasing knowledge on the biology and availability of new combination treatments have changed the management of PC. There is now emerging consensus that a subgroup of patients, previously considered poor candidates for resection may benefit from surgical approach, particularly when preceded by preoperative therapy.

New treatment options, such as FOLFIRINOX [8] or gemcitabine/nab-paclitaxel [9], have resulted in new clinical outcomes that could result in improved prognoses. The FOLFIRINOX regimen has provided survival benefits for patients with metastasized and locally advanced PC. Moreover, the increase in objective response rate can also increase the number of resectable patients. A recent meta-analysis [10] showed a good down-staging after neoadjuvant FOLFIRINOX-based therapy in patients with borderline resectable/unresectable PC, with a total R0 resection rate of 39.4\%. Specifically, among borderline resectable PC, R0 resection was possible in $63.5 \%$ (95\% CI, 49\%$76 \%)$ of the cases, while the rate of R0 resection was $22.5 \%(95 \% \mathrm{CI}, 13.3-35.4)$ in unresectable PC. 
As expected, more intensive therapies are associated with increased toxicities.

In order to improve prognosis without increasing toxicities or negatively impact quality of life, we started a trial exploring the anticancer properties of Meriva ${ }^{\circledR}$ associated with gemcitabine as first line treatment in advanced PC.

Here we present a case of a patient with unresectable disease, confirmed at first surgical exploration, who obtained late response after initial stable disease. Our patient received 14 cycles of therapy before achieving a radiological partial response. Our patient is disease free at 9 month from surgery and 28 months from initial diagnosis and he still is in good clinical conditions.

Moreover, the results was obtained with a minimally toxic regimen. Treatment tolerance was excellent and chemotherapy had no negative impact on quality of life. Preliminary results of our aforementioned trial [11] are positive as regards the primary end point and further analysis are ongoing to evaluate the correlation with serum biomarkers.

The innovative combination of gemcitabine and Meriva ${ }^{\circledR}$ represents an attempt to find effective but less toxic treatment strategies for PC. In particular, this is the first report of the success in achieving radical resection after single agent therapy combined with oral curcumin supplement.

\section{References.}

[1] M. Malvezzi, P. Bertuccio, F. Levi, C. La Vecchia, E. Negri. European cancer mortality predictions for the year 2013. Ann Oncol. 24 (2013) 792-800.

[2] G. Barugola, S. Partelli, S. Marcucci, N. Sartori, P. Capelli, C. Bassi, P. Pederzoli, M. Falconi. Resectable pancreatic cancer: who really benefits from resection? Ann Surg Oncol. 16 (2009) 3316-3322.

[3] G.R. Varadhachary, E.P. Tamm, J.L. Abbruzzese, H.Q. Xiong, C.H. Crane, H. Wang, J.E. Lee, P.W. Pisters, D.B. Evans, R.A. Wolff. Borderline resectable pancreatic cancer: definitions, management, and role of preoperative therapy. Ann Surg Oncol. 13 (2006) 10351046.

[4] M. Glanemann, B. Shi, F. Liang, X.G. Sun, M. Bahra, D. Jacob, U. Neumann, P. Neuhaus. Surgical strategies for treatment of malignant pancreatic tumors: extended, standard or local surgery? World J Surg Oncol 6 (2008) 123.

[5] J.M. Laurence, P.D. Tran, K. Morarji, G.D. Eslick, V.W. Lam, C. Sandroussi. A systematic review and meta-analysis of survival and surgical outcomes following neoadjuvant chemoradiotherapy for pancreatic cancer. J Gastrointest Surg.15 (2011) 2059-2069.

[6] A. Andriulli, V. Festa, E. Botteri, M.R. Valvano, M. Koch, C. Bassi, P. Maisonneuve, P.D. Sebastiano. Neoadjuvant/preoperative gemcitabine for patients with localized pancreatic cancer: a meta-analysis of prospective studies. Ann Surg Oncol. 19 (2012) 1644-1662.

[7] A. Shehzad, F. Wahid, Y.S. Lee. Curcumin in cancer chemoprevention: molecular targets, pharmacokinetics, bioavailability, and clinical trials. ArchPharm (Weinheim). 343 (2010) 489-499.

[8] T. Conroy, F. Desseigne, M. Ychou, O. Bouché, R. Guimbaud, Y. Bécouarn, A. Adenis, J.L. Raoul, S. Gourgou-Bourgade, C. de la Fouchardière, J. Bennouna, J.B. Bachet, F. KhemissaAkouz, D. Péré-Vergé, C. Delbaldo, E. Assenat, B. Chauffert, P. Michel, C. Montoto-Grillot, M. Ducreux; Groupe Tumeurs Digestives of Unicancer; PRODIGE Intergroup. et al. FOLFIRINOX versus gemcitabine for metastatic pancreatic cancer. N Engl J Med. 364 (2011) 1817-1825. 
[9] D.D.Von Hoff, T. Ervin, F.P. Arena, E.G. Chiorean, J. Infante, M. Moore, T. Seay, S.A. Tjulandin, W.W. Ma, M.N. Saleh, M. Harris, M. Reni, S. Dowden, D. Laheru, N. Bahary, R.K. Ramanathan, J. Tabernero, M. Hidalgo, D. Goldstein, E. Van Cutsem, X. Wei, J. Iglesias, M.F. Renschler. Increased survival in pancreatic cancer with nab-paclitaxel plus gemcitabine. N Engl J Med. 369 (2013) 1691-1703.

[10] F. Petrelli, A. Coinu, K. Borgonovo, M. Cabiddu, M. Ghilardi, V. Lonati, E. Aitini, S. Barni; Gruppo Italiano per lo Studio dei Carcinomi dell'Apparato Digerente (GISCAD). FOLFIRINOX-based neoadjuvant therapy in borderline resectable or unresectable pancreatic cancer: a meta-analytical review of published studies. Pancreas. 44 (2015) 515-21.

[11] C. Soldà, R. Bardini, C. Sperti, G. Da Dalt, M. Gion, P. Fiduccia, F. Ursini, C. Aliberti, D. Pastorelli. Phase II study of gemcitabine and curcumin as first line treatment for locally advanced or metastatic pancreatic cancer: preliminary data Ann Oncol 26 (suppl 4) (2015) iv10. 